Relations industrielles

Industrial Relations

\title{
Dandurand, Pierre, dir., Enjeux actuels de la formation professionnelle
}

\section{Marie-Thérèse Chicha}

Volume 49, numéro 4, 1994

Syndicats et restructuration économique

Unions and Economic Restructuring

URI : https://id.erudit.org/iderudit/050980ar

DOI : https://doi.org/10.7202/050980ar

Aller au sommaire du numéro

Éditeur(s)

Département des relations industrielles de l'Université Laval

ISSN

0034-379X (imprimé)

1703-8138 (numérique)

Découvrir la revue

Citer ce compte rendu

Chicha, M.-T. (1994). Compte rendu de [Dandurand, Pierre, dir., Enjeux actuels de la formation professionnelle]. Relations industrielles / Industrial Relations, 49(4), 858-861. https://doi.org/10.7202/050980ar

Tous droits réservés (C) Département des relations industrielles de l'Université Laval, 1994
Ce document est protégé par la loi sur le droit d'auteur. L'utilisation des services d'Érudit (y compris la reproduction) est assujettie à sa politique d'utilisation que vous pouvez consulter en ligne.

https://apropos.erudit.org/fr/usagers/politique-dutilisation/ 
Enjeux actuels de la formation professionnelle, sous la direction de Pierre Dandurand. Question de culture ${ }^{\circ} 19$, Québec, Institut québécois de recherche sur la culture, 1993, 271 p., ISBN 2-89224-189-8.

Ce livre qui rassemble les contributions de divers auteurs, sous la direction de Pierre Dandurand, a pour objectif d'analyser les questions qui se posent en matière de formation professionnelle dans un contexte de restructuration économique. À cette fin, la formation professionnelle est envisagée comme un continuum qui se déroule en plusieurs étapes interreliées et où interviennent divers acteurs, notamment l'école, l'entreprise et l'État.

Dans le premier chapitre, Christian Payeur et Roland Ouellet examinent la formation professionnelle au secondaire, à partir du Plan d'action de 1986. Après avoir situé cette réforme dans son contexte historique, ils présentent ses principales dispositions et tentent d'en évaluer l'impact à travers les statistiques disponibles. Ils indiquent alors comment les différences de définitions et de méthodologie limitent tout exercice d'évaluation et mettent ainsi en garde contre une interprétation rapide des données statistiques disponibles. Le point fort de la réforme de 1986 est d'avoir intégré les nouvelles technologies dans le contenu des cours qui, par ailleurs, sont devenus strictement axés sur la formation professionnelle. Par contre, la hausse des seuils d'accès, dont ces auteurs reconnaissent le bien-fondé, a eu des effets d'exclusion notamment sur les jeunes n'ayant pas une bonne formation académique et sur les adultes peu scolarisés. Cet effet négatif traduit l'absence d'un projet social plus vaste dans lequel aurait dû s'inscrire cette réforme.

Dans le deuxième chapitre, Madeleine Gauthier analyse la situation des jeunes qui ont laissé l'école secondaire pour entrer sur le marché du travail. Elle êvalue la relation entre scolarisation et emploi chez les jeunes à partir de l'Enquête longitudinale sur l'activité de Statistique Canada effectuée en 1988-1989. Elle souligne qu'il y a une pluralité de facteurs expliquant la faible scolarisation : déficiences physiques ou mentales, difficultés d'apprentissage, conditions socio-économiques, grossesse. Son analyse fait ressortir la tendance à une «dualisation des situations face à l'emploi, dualisation entre les groupes d'âges qui vient amplifier les écarts à l'intérieur du même groupe d'âge. Le fait d'être jeune, s'il est accompagné d'un faible niveau de scolarité, pourrait caractériser aujourd'hui la catégorie sociale la plus vulnérable. [...] On retrouverait à un extrême les travailleurs stables, le plus souvent chez les aînés, et à l'autre, les travailleurs précaires, plus nombreux chez les jeunes et en plus forte proportion chez les moins scolarisés qui sont plus jeunes ». L'auteure attire l'attention sur un groupe particulièrement vulnérable, les décrocheuses pour motif de grossesse, et sur la nécessité d'une large concertation destinée à leur permettre de devenir actives sur le marché du travail.

Dans le troisième chapitre, Pierre Doray et Lorraine Rochon se demandent si les nouvelles initiatives en matière de formation professionnelle dans les cégeps représentent un changement dans les relations formation-travail. Après avoir analysé la question de la pertinence des programmes vis-à-vis des caractéristiques et des besoins du nouveau marché du travail et les nombreux débats qu'elle soulève, les auteurs examinent deux initiatives qui pourraient représenter de nouvelles relations formation-travail : les centres spécialisés et la formation sur mesure en établissement. Leur analyse indique qu'il y a une tendance à un « repositionnement » de la formation professionnelle dans 
les cégeps, notamment par le biais des nouveaux rôles sociaux des employeurs et des syndicats, de l'association entre enseignement et recherche appliquée et enfin de la prise en compte des dynamiques locales et régionales.

Le quatrième chapitre, par Renée Cloutier et Claude Trottier, traite de la formation universitaire de premier cycle et de son effet sur l'insertion professionnelle. Les auteurs analysent les modes d'insertion professionnelle des diplômés à l'aide de différentes mesures du taux de placement. Deux résultats importants ressortent de leur étude : le premier est qu' « en dépit du taux de placement relativement très élevé des personnes diplômées de l'université, la situation d'une proportion non négligeable d'entre elles demeurait problématique dans la mesure où elles n'avaient pas encore accédé, deux ans après leur sortie de l'université, à un emploi permanent relié à leur domaine d'études "; le second résultat est que l'appartenance sociale des diplômés n'a pas d'effet direct sur leur insertion professionnelle mais que « son effet semble s'exercer par l'intermédiaire du champ d'études vers lequel elles se sont orientées ».

Dans le cinquième chapitre, Diane-Gabrielle Tremblay se penche sur la question des besoins de formation compte tenu de l'évolution économique et de l'innovation. Les économies de la plupart des pays industrialisés de « longue date » ont atteint une certaine maturité qui les obligent à évoluer vers de nouvelles productions, de haute qualité. Une telle évolution requiert toutefois que l'on augmente le niveau de formation et de qualification de la main-d'œuvre et pour ce faire, il est impérieux que l'État intervienne en tenant compte des particularités et de la position concurrentielle de chacun des secteurs d'activités. L'auteure développe son argumentation autour de trois axes majeurs : en premier lieu, l'effet du contexte économique actuel sur les types de production et, par ricochet, les techniques de production et le travail lui-même; en deuxième lieu, l'état de la situation en matière de formation dans les entreprises québécoises, notamment le constat selon lequel cette formation est orientée vers un modèle de minimisation de coûts à court terme plutôt que d'innovation, de qualité et d'investissement. Enfin, la nécessité d'une intervention de l'État qui devrait aider « les entreprises à évoluer vers une meilleure qualification des emplois en mettant en œuvre une politique cohérente d'emploi et de développement des ressources humaines ».

Le sixième chapitre, par Colette Bernier, s'intitule «L'entreprise et le couple qualification/formation: une articulation de plus en plus étroite et névralgique?». L'auteure présente une analyse centrée sur la formation en entreprise et les liens entre cette formation et celle acquise en milieu scolaire. Selon elle, la compétitivité amène les entreprises à multiplier les changements technologiques et organisationnels modifiant les enjeux de la formation; elles se sont ainsi donné de nouveaux critères de qualité et de diversité de leurs produits et de leurs services. Ces critères interpellent non seulement les connaissances des salariés mais également leurs attitudes et comportements. Des études de cas effectuées dans deux secteurs - la finance et la chimie/pétrochimie - lui permettent de dégager deux axes principaux d'innovations de formation : un premier axe concerne le renouvellement de la formation comme telle, sur la base de la socialisation des salariés et de leur responsabilisation à l'égard de la qualité du produit; le deuxième axe concerne l'établissement de nouveaux liens école-entreprise sur la base d'une requalification de certains personnels. 
Dans le chapitre sept, Marc-André Deniger et Monique Provost examinent la politique d'employabilité qui est devenue le leitmotiv de l'aide sociale depuis la réforme de 1989. Après avoir présenté les différents volets du nouveau système d'aide sociale, ils examinent les rares évaluations qui en ont été faites jusqu'ici, évaluations dont les résultats indiquent les limites majeures de la politique d'employabilité en matière d'insertion sur le marché du travail. Une des explications réside dans le fait que, de façon tout à fait paradoxale, le développement de l'employabilité est conçu en fonction des clientèles qui en ont le moins besoin. De plus, cette notion met l'accent sur la notion de responsabilisation des individus et sur leurs caractéristiques tout en négligeant l'état du marché du travail; ce dernier qui se caractérise par un taux de chômage élevé rend illusoire une insertion réelle sur le marché du travail des clientèles les plus démunies. Les auteurs situent également la réforme de l'aide sociale dans le contexte de restrictions budgétaires et concluent que l'objectif de réduction des dépenses sociales a dû constituer un enjeu majeur de cette réforme.

Dans le huitième chapitre, Pierre Paquet traite de «l'évolution des politiques canadiennes et québécoises de formation des adultes depuis 1960 ». L'auteur brosse un portrait exhaustif de l'évolution et du développement des politiques étatiques de formation de la main-d'œuvre au cours des trente dernières années. Pour ce faire, il a recours à de nombreux mémoires et énoncés produits par différents intervenants concernés par la question : comités d'experts, fonctionnaires, associations patronales et syndicales de la scène provinciale et fédérale. S'appuyant sur certaines données historiques, l'auteur tente de démontrer que les changements récents dans les politiques gouvernementales renforcent et perpétuent la création d'une société duale.

En conclusion du livre, Pierre Dandurand met en relief les principaux points abordés par les différents auteurs. Il articule sa synthèse autour de deux éléments. En premier lieu, trois grands axes autour desquels tend à se réorganiser le champ de la formation professionnelle : l'axe des changements structurels de l'économie, celui de la revalorisation de la formation professionnelle et celui de la place nouvelle et grandissante des entreprises dans la formation professionnelle. En deuxième lieu, deux défis auxquels devront répondre les politiques de formation professionnelle, soit l'insertion professionnelle des jeunes et la réinsertion des travailleurs sans emploi ainsi que les risques de dualisation croissante de la société. Il termine en soulignant l'enjeu majeur de l'évolution future de la formation professionnelle, soit la conciliation de l'économique et du social, de l'efficacité et de l'équité.

Comme on peut le constater, ce livre ne néglige aucune des dimensions essentielles de la formation professionnelle. De plus, à travers la plupart des chapitres on retrouve deux traits importants : premièrement, la présentation d'informations relatives à l'historique et au contenu des politiques et programmes de formation étudiés, ce qui permet de mieux se situer dans un domaine où la multiplication des programmes et l'enchevêtrement des juridictions constituent un véritable casse-tête; deuxièmement, une double perspective analytique des programmes de formation professionnelle qui sont examinés aussi bien en termes d'efficacité que d'équité, contrastant ainsi avec la plupart des analyses actuelles centrées sur des questions de productivité et de compétitivité économiques. Ce livre, d'accès facile, s'adresse aussi bien aux chercheurs qu'aux décideurs en matière de formation professionnelle; de plus, plusieurs de ses articles peuvent 
constituer des lectures intéréssantes dans le cadre de cours relatifs à la formation professionnelle ou à l'analyse du marché du travail.

Marie-Thérèse CHICHA

Université de Montréal

Profit Sharing: Does it Make a Difference? by Douglas L. KRUSE, Kalamazoo, Mich., The W.E. Upjohn Institute for Employment Rechearch, 1993, 289 p., ISBN 0-88099-138-0 and ISBN 0-88099-137-2 (pbk).

The purpose of this book is to analyze the existing empirical evidence on the impact of profit sharing on company performance and employment stability, while adding the results of a comprehensive empirical study conducted by the author himself. The question posed by the book title is an important one, as companies increasingly turn to profit sharing as a means of improving their performance, and social policy analysts argue for profit sharing as a means of preserving employment and enhancing employment stability.

Does profit sharing really do either? Kruse tackles this question in five chapters. The first chapter defines the concept and discusses its recent growth and current prevalence in the United States. The second chapter extends this analysis by examining the specific motives that companies have for adopting profit sharing, and identifies the types of firms and circumstances under which profit sharing is likely to be implemented.

Chapters 3 and 4 are the real meat of this book, as they focus, in turn, on the two main issues of interest - productivity and employment stability. Chapter 3 begins by examining the existing evidence linking profit sharing to company productivity. Previous studies suggest such a link but the causal direction is not clear. Furthermore, most of the existing studies do not deal with the possibility that profit sharing firms may have other policies that are in fact responsible for increased productivity.

Based on longitudinal study of 250 U.S. firms that have profit sharing, and 250 that do not, and controlling for possible confounding variables, Kruse concludes that profit sharing can lead to improved company productivity, but this is dependent on the nature of the plan and size of firm. For example, plans that base their payout on a percentage of wages show no favourable effects (which is just what theory would suggest, since these are not true profit sharing plans). In terms of firm size, profit sharing was most effective in small firms, but was also effective in very large firms. However, it is not clear whether this latter result is due to firm size per se, or to a difference in the nature of profit sharing plans across the different size categories of firms.

Chapter 4 examines the evidence on whether, by reducing the fixed component of labour costs, profit sharing can enhance employment stability within firms, as Weitzman has argued (The Share Economy, Cambridge, Harvard University Press, 1994). Of course, this argument assumes that profit sharing substitutes, to some extent at least, for fixed wages, which is not at all clear. Historically, most proponents of profit sharing have argued that it should serve as a bonus on top of competitive wages. 